\title{
Peridynamic Simulations of Nanoindentation Tests to Determine Elastic Modulus of Polymer Thin Films
}

\author{
Emrah Celik ${ }^{a^{*}}$, Erkan Oterkus ${ }^{b}$, Ibrahim Guven $^{c}$ \\ ${ }^{a}$ Mechanical and Aerospace Engineering Department, University of Miami, \\ Coral Gables, Florida, USA \\ ${ }^{\mathrm{b}}$ Department of Naval Architecture, Ocean and Marine Engineering, \\ University of Strathclyde, Glasgow, United Kingdom \\ ${ }^{\mathrm{c}}$ Department of Mechanical and Nuclear Engineering, \\ The Virginia Commonwealth University, Richmond, Virginia, USA \\ * E-mail: e.celik@miami.edu, Phone: 1-305-284-9364
}

\begin{abstract}
This study combines atomic force microscope (AFM) nanoindentation tests and peridynamic (PD) simulations to extract the elastic moduli of polystyrene (PS) films with varying thicknesses. AFM nanoindentation tests are applied to relatively hard PS thin films deposited on soft polymer (polydimethylsiloxane (PDMS)) substrates. Linear force versus deformation response was observed in nanoindentation experiments and numerical simulations since the soft PDMS substrate under the stiff PS films allowed bending of thin PS films instead of penetration of AFM tip towards the PS films. The elastic moduli of PS
\end{abstract}


thin films are found to be increasing with increasing film thickness. The validity of both the simulation and experimental results is established by comparison against those previously published in the literature.

Keywords; Atomic Force Microscopy (AFM), Nanoindentation, Peridynamic Theory, Elastic Moduli

\section{Introduction}

Polymer thin films can significantly alter the properties of surfaces such as corrosion resistance, wettability, adhesion, biocompatibility, morphology, conductivity, etc. Hence, these films are employed in many applications in nanotechnology, automotive, biomedicine and energy conversion. The properties and the performance of these films are correlated to the film geometry, film chemical composition, and surface properties. Among these, mechanical properties are particularly important which determine reliable use of these materials systems under different types of loading conditions.

Several mechanical characterization techniques have been introduced in the literature for mechanical characterization of thin films such as wrinkling method $[1,2]$, resonance method [3, 4] and nanoindentation [5-10]. Among all, nanoindentation is the most commonly used technique for mechanical characterization of thin films due to the ease of the experiment. Atomic force microscope (AFM) can be utilized for nanoindentation of soft thin films [8-10] and nanoindenter is preferred for the characterization of hard thin films [5-7]. However, as the thin film thickness becomes very small, the resolution of the equipment for the obtainable indentation depth becomes critical, and it determines the minimum thickness 
of the film that can be tested. Also, the effect of the substrate underneath the thin film becomes significant. Therefore, accurate extraction of mechanical properties of the film depends on nature of the substrate modeling. The development of a new methodology becomes essential for mechanical characterization of ultra-thin films.

Although nanoindentation of ultra-thin films may pose challenges because of their thickness limitation, these films can be tested under bending loads if deposited on soft substrates without any limitation on the thickness. Bending tests of thin films can be performed by using an AFM which is preferred over the traditional nanoindenters because it permits the application of extremely small indentation forces through the tip of the AFM cantilever, thus leading to truly nanometer scale deformations. However, the extraction of the mechanical properties requires accurate characterization of the soft substrate underneath the film and modeling of the bending experiment.

Finite element analysis coupled with nanoindentation experiments have been used to evaluate/extract material properties of the thin films previously [11-14]. For ultra-thin films having nano-scale thickness, the commonly accepted simulation technique in the literature is Molecular Dynamics Simulations (MDS). In MDS, each individual atom is modeled, and a suitable interatomic potential is used in order to define the interaction between the atoms of the nanostructure. However, modeling each atom proves to be extremely demanding from a computational standpoint [15]. An alternative to MDS is peridynamics which can be considered as the continuum version of the MDS. Therefore, this study utilizes the Peridynamic theory introduced by Silling [16]. Peridynamics is a version of the non-local theory which was first introduced by Eringen and Edelen [17] and Kroner [18]. Peridynamics 
has been applied for the solution of many different problems and material systems including crack branching [19], plasticity [20], viscoelasticity [21], viscoplasticity [22], composite materials [23, 24], nanowires [25], bounded and unbounded domains [26] etc.

This study presents a combined experimental and computational approach for determining the elastic modulus of relatively stiff ultra-thin films deposited on soft substrates. An in-house AFM is used for the mechanical tests and Peridynamic theory for the numerical simulations. The next two sections describe the experimental setup and a description of the PD theory. Subsequently the experimental data and numerical simulation results are compared in order to extract mechanical properties of the thin films.

\section{Experimental Details}

\subsection{Specimen preparation}

Two different types of samples are prepared: (i) polydimethylsiloxane (PDMS) in the form of bulk sample, and (ii) thin-film polystyrene (PS) deposited on the bulk PDMS. The specimen configurations of bulk PDMS and thin PS film are shown in Fig. 1A. The thickness of the bulk PDMS is approximately $5 \mathrm{~mm}$ while the PS film thickness is varied between 700 $\mathrm{nm}$ to $10 \mu \mathrm{m}$.

The PDMS samples are prepared using a commercially available silicone encapsulant kit (Sylgard 184). Resin is first mixed with the curing agent (10:1 mass ratio) manually with a glass stirring rod for approximately 15 minutes and then the mixture is poured over a precleaned surface of a silicon wafer. PDMS is then degassed by placing it in a vacuum chamber and subjecting it to pressures below $\sim 1000$ millitorr for 3 to 5 minutes. The curing is 
completed at atmospheric pressure at $50-60^{\circ} \mathrm{C}$ for 4 to 5 hours. After the curing is completed, PDMS sample is peeled off from the wafer surface. The wafer-contacted side of the PDMS is used in the nanoindentation experiments due to the low roughness of the surface.

The thin-film PS samples are prepared by spin coating of PS on bulk PDMS substrate. Solid polystyrene particles are first dissolved in toluene. The solutions with different PS concentrations ( $2 \%$ to $10 \mathrm{wt} \%)$ are prepared in order to achieve different PS film thicknesses. PS solutions are then spin coated on PDMS substrate at a spin velocity of 3000 revolution per minute. The thickness of the PS film is measured to be between $700 \mathrm{~nm}$ to $10 \mu \mathrm{m}$ using AFM. The SEM micrograph of the thickest film on PDMS surface is shown in Fig.1B. Roughness and thickness of PS and PDMS surfaces are characterized by AFM imaging (Fig.2). Fig.2A shows the smooth surface of wafer-contacted side of the PDMS specimen where the roughness is measured to be less than $50 \mathrm{~nm}$ using the AFM. Fig.2B shows the thickness measurement using AFM where the step height between the PDMS (dark, lower side) and PS (higher, pink side) surfaces represents the thickness of PS films.

\subsection{AFM nanoindentation tests}

Both the bulk PDMS and PS thin film specimens are indented with the tip of a vendorcalibrated Mikromasch ultra-sharp cantilevers as shown in Fig.3A. Raw piezo displacement versus cantilever deflection data are converted into force versus indentation response using standard methodology as described earlier [27]. Briefly, cantilever stiffness is multiplied by the cantilever deflection to determine the applied force on the surface. Total indentation on the sample is equal to the difference between the rigid sample and actual (PS/PDMS) sample 
deflection response as denoted in Fig.3B. The repeatability of the experimental tests is established by performing the indentation test three consecutive times on the each type of sample.

Simulations of the AFM indentation experiments are performed by employing the Peridynamic theory in search of a material constant that yields the best fit to the measured force-deformation relation. While treating the tip of the AFM as a rigid indenter, this inverse approach permits the extraction of the elastic moduli of PS polymer films.

\subsection{Peridynamic formulation}

In this study, bond-based peridynamics is utilized for computational analysis. According to bond-based peridynamics, material points are interacting with each other in a non-local manner. The interaction (bond) forces between material points are assumed as pairwise, equal and opposite to each other. It is also assumed that there is no interaction between material points if the distance between them is greater than a specific distance (horizon). During the solution process, stretch of each bond is monitored and if the stretch value exceeds a critical stretch value, then the bond is considered to be broken as shown in Figure 4A. Peridynamic equations are always valid regardless of the broken bonds. The damage parameter for each material point can be simply defined as the ratio of the number of broken bonds and the number of bonds in the undeformed configuration associated with that material point. Therefore, it is straightforward to represent damage as opposed to classical continuum mechanics based approaches. Moreover, in peridynamics, it is possible to specify different properties to bonds representing interfaces between different materials with respect to their bulk material properties. 
In bond-based peridynamics, the equation of motion can be expressed as

$$
\rho(\mathbf{x}, t) \frac{\partial^{2} u(\mathbf{x}, t)}{\partial t^{2}}=\int_{H_{\mathbf{x}}} \mathbf{f}\left(\mathbf{u}\left(\mathbf{x}^{\prime}, t\right)-\mathbf{u}(\mathbf{x}, t), \mathbf{x}^{\prime}-\mathbf{x}, t\right) d V_{\mathbf{x}^{\prime}}+\mathbf{b}(\mathbf{x}, t)
$$

where $\rho$ and $\mathbf{u}$ represent the density and the displacement of the material point, $t$ denotes time, $\mathbf{f}$ is the bond force between material points $\mathbf{x}$ and $\mathbf{x}^{\prime}$ (see Figure 5), and $H_{\mathbf{x}}$ is the horizon of the material point $\mathbf{x}$. For an isotropic material, the bond force can defined as (see Figure 4B)

$$
\mathbf{f}\left(\mathbf{u}\left(\mathbf{x}^{\prime}, t\right)-\mathbf{u}(\mathbf{x}, t), \mathbf{x}^{\prime}-\mathbf{x}, t\right)= \begin{cases}\frac{\xi+\eta}{|\xi+\eta|}(c s), \text { if } s<s_{0} \\ 0, \text { if } s \geq s_{0}\end{cases}
$$

in which $\xi$ is the bond vector, i.e. $\mathbf{x}^{\prime}-\mathbf{x}, \eta$ represents the relative displacement between two material points, i.e. $\mathbf{u}\left(\mathbf{x}^{\prime}, t\right)-\mathbf{u}(\mathbf{x}, t)$, and stretch can be defined as $s=(|\xi+\eta|-|\xi|) /|\xi|$. Moreover, the bond constant, $c$ can be expressed in terms of Young's modulus of the material and the horizon size, $\delta$, as [28]

$$
c=\frac{12 E}{\pi \delta^{4}}
$$

\subsection{Peridynamic contact analysis}

In this study, the indenter is assumed as a rigid structure. Based on the peridynamic contact analysis approach for a rigid indenter presented in [29], at each time step of the simulation process, unphysical penetration of the indenter inside the target material should 
be prevented. If such situation occurs, material points inside the indenter are moved to closest locations outside of the indenter surface (refer to Figure 6). As a result of this relocation, the modified velocity of the material point $i$ at $t+\Delta t, \bar{V}_{i}^{t+\Delta t}$, can be calculated as

$$
\bar{V}_{i}^{t+\Delta t}=\frac{\left(\bar{u}_{i}^{t+\Delta t}-u_{i}^{t}\right)}{\Delta t}
$$

where $\overline{\mathbf{u}}_{i}^{t+\Delta t}$ is the modified displacement of the material point $i$ at $t+\Delta t, \mathbf{u}_{i}^{t}$ is the displacement of the material point $i$ at $t$, and $\Delta t$ is the time increment. As a result of the contact between indenter and target material, a reaction force occurs. The contribution of the material point $i$ to the total reaction force at time $t+\Delta t, F_{i}^{t+\Delta t}$, can be defined as

$$
F_{i}^{t+\Delta t}=-1 \times \rho_{i} \frac{\left(\bar{v}_{i}^{t+\Delta t}-V_{i}^{t+\Delta t}\right)}{\Delta t} V_{i}
$$

where $V_{i}^{t+\Delta t}$ is the velocity of the material point $i$ at time $t+\Delta t$ before relocating the material point $i, \rho_{i}$ and $V_{i}$ represent the density and volume of the material point $i$, respectively.

Finally, the total reaction force on the indenter at time $t+\Delta t, F^{t+\Delta t}$, can be obtained by summing up the contributions of all material points inside the indenter as,

$$
F^{t+\Delta t}=\sum_{i=1}^{N} F_{i}^{t+\Delta t} \lambda_{i}^{t+\Delta t}
$$

where

$$
\lambda_{i}^{t+\Delta t}=\left\{\begin{array}{l}
1, \text { if the point } i \text { is inside the indenter } \\
0, \text { if the point } i \text { is outside the indenter }
\end{array}\right.
$$

and $N$ is the total number of material points in the target material. 


\section{Results}

Force versus deformation measurements for bulk PDMS and thin PS films deposited on PDMS are shown in Fig. 7. The PS film thickness is varied as $700 \mathrm{~nm}, 1100 \mathrm{~nm}, 3100$ $\mathrm{nm}$ and $10000 \mathrm{~nm}$. The representative force versus deformation data is obtained through averaging of the repeated tests with the usage of error bars. As observed in this figure, force changes nonlinearly as a function of sample deformation during the indentation of bulk PDMS suggesting a significant change in contact area between the AFM tip and the soft PDMS material. However, testing of all four PS thin films deposited on PDMS results in linear force-deformation variations. This is a clear indication that the deformation mode of hard PS thin film is different than that of soft bulk PDMS and that the deformation of hard PS thin film is dominated by film bending instead of indentation. Fig. 7 also indicates that as the thin film thickness decreases, the amount of force to deform the sample decreases due to the decrease in bending rigidity.

In order to validate the numerical simulation accuracy, $\mathrm{PD}$ analysis is first performed to model the measured force-deformation response of the bulk PDMS specimen. In this analysis, the following simulation parameters are used: total of 500,000 material points, grid spacing of $d=200 \mathrm{~nm}$ and horizon radius of $\delta=603 \mathrm{~nm}$. The indenter velocity is $v_{0}=20 \mathrm{~m} / \mathrm{s}$. While searching for the elastic modulus, a simple optimization algorithm is used to minimize the difference between the measured and computed force-indentation depth relations. The elastic modulus resulting the best correlation was extracted as the correct elastic modulus for the material. Fig. 8 shows the measured force-indentation depth and its PD simulation using the extracted value of elastic modulus. The correlation between the 
measurement and simulation is remarkable. In this analysis, PDMS elastic modulus is extracted as 10MPa which compares well with the previously published studies for PDMS $[30]$.

Next, the PD simulation of indentation experiment involving thin-film PS deposited on PDMS is performed. For PDMS material modulus, the extracted value from the previous simulation is used. In addition, the same simulation parameters of the PDMS analysis are used for the PD analysis for consistency in results. However, indenter velocity is reduced to is $v_{0}=10 \mathrm{~m} / \mathrm{s}$ due to smaller thickness of PS films compared to the bulk PDMS. The comparison of force-deformation response obtained from the PD simulation against the measurements for the PS film with a thickness of $1100 \mathrm{~nm}$ is shown in Fig. 9, which exhibits a very good agreement. PD simulations confirm the linear material response of deposited PS films measured in the tests.

The PD simulation of the deformed shape of thin PS film deposited on PDMS substrate is shown in Fig. 10. It is clear that the deformation pattern, unlike the PDMS case, is a combination of local indentation and global bending of the PS layer. Indentation pattern at the top surface of the PS film under the indenter is visible in the inlet of Figure 10B. The tip indentation in the vicinity of the indenter is present and the bending of the PS thin film and deformation of the bottom surface of the thin film is clearly visible. The deformed shape of the PS thin film deposited on soft PDMS substrate in conjunction with the linear forcedeformation response obtained by experiments and PD simulations clearly indicate the bending behavior of the relatively hard PS films. 
The elastic moduli of the PS films were extracted in comparison with AFM experiments and PD simulations. The variation of elastic modulus as a function of film thickness is given in Fig. 11. The figure denotes that the elastic modulus shows an increasing trend as the thickness of the film increases. This variation is consistent with the measurements of Overney et al. [31] and observations of Teichroeb and Forrest [32] arguing that the thinfilm polystyrene is less glassy than the bulk samples. Elastic modulus linearly increased as the film thickness increased. We expect that, as the thickness increases, the thickness effect will vanish and the elastic modulus of PS specimens will approach to the bulk modulus of PS which is reported as $3 \mathrm{GPa}$ [33]. In literature, similar behavior of thin film modulus increase as a function of film thickness and its saturation to the bulk material modulus were commonly observed. The saturation thickness at which the film modulus equalized to that of the bulk value was found to be lower than 200 nanometers $[34,35]$ in some studies. However, similar to our study, continuous increase of elastic modulus as the thickness exceeded 500 nm was observed in other studies as well $[36,37]$. The change of elastic modulus in some of these studies were shown to be linearly increasing [37], bi-linear [36] and nonlinear [34, 35] as a function of film thickness.

\section{Conclusions}

A new technique for extracting elastic moduli of ultra-thin films based on a combined experimental and computational method is demonstrated. The stiff/hard material is deposited on a soft substrate with known material properties. Combined bending/indentation 
deformation of the material system allows accurate AFM measurements and effective extraction of material properties via peridynamic theory. Unlike the conventional indentation experiments, testing of stiff films deposited on soft substrates is not limited by sample thickness and that mechanical properties of ultra-thin films can be reliably characterized.

The force-deformation measurements of the bulk PDMS and PS thin-film specimens deposited on bulk PDMS substrates are compared against the PD simulations to extract mechanical properties of bulk PDMS and PS thin films. The extracted values for PDMS and thin-film PS are consistent with the previous research from the literature. Both experiments and simulations clearly indicate that deformation type is indentation for soft PDMS substrates but it occurs via bending on relatively hard PS films deposited on soft PDMS substrates.

\section{References}

1. R. Huang, C. M. Stafford, and B. D. Vogt, Materials Research Society Symposia Proceedings 924 (2009).

2. C. M. Stafford, C. Harrison, K. L. Beers, A. Karim, E. J. Amis, M. R. Vanlandingham, H. C. Kim, W. Volksen, R. D. Miller and E. E. Simonyi, Nature Materials 3 (2004).

3. S. A. Jade, and J. G. Smits, IEEE Transactions on Ultrasonics, Ferroelectrics, and Frequency Control 46-4 (1999) 768.

4. L. Kiesewetter, J.-M. Zhang, D. Houdeau and A. Steckenborn, Sensors and Actuators A 35 (1992) 153.

5. Y-G. Jung, B. R. Lawn, M. Martyniuk, H. Huang and X. Z. Hu, Journal of Materials Research 19-10 (2004) 3076. 
6. D. Son, Y-H. Lee, J-H.Ahn, and D. Kwon, Materials Research Society Symposia Proceedings 562 (1999) 201.

7. S.H. Hong, K.S. Kim, Y.-M. Kim, J.-H. Hahn, C.-S. Lee, and J.-H. Park, Composites Science and Technology 65 (2005) 1401.

8. J. Domke, and M. Radmacher, Langmuir 14 (1998) 3320.

9. L. Sirghi, A. Ruiz, P. Colpo and F. Rossi, Thin Solid Films 517 (2009) 3310.

10. K. Miyake, N. Satomi, and S. Sasaki, Applied Physics Letters 89 (2006) 31925.

11. J.A.Knapp, D.M.Follstaedt, J.C.Barbour, S.M.Myers, Nuclear Instruments and Methods in Physics Research Section B: Beam Interactions with Materials and Atoms, 127, (1997), pp. 935-939

12. K. J. Van Vliet and A. Gouldstone, Surface Engineering, 17, (2001), pp.140-5

13. L.GanB.Ben-Nissan, Computational Materials Science, 8, (1997),pp. 273-81

14. D. E. Ojos, J. Sort, Ch16, In Book: Applied Nanoindentation in Advanced Materials, (2017) pp.369-391

15. P. Walsh, A. Omeltchenko, R. K. Kalia, A. Nakano, and P. Vashishta, Applied Physics Letters 82-1 (2003) 118.

16. S. A. Silling, Journal of the Mechanics and Physics of Solids 48 (2000) 175.

17. A. C. Eringen, and D. G. B. Edelen, International Journal of Engineering Science 10-3 (1972) 233.

18. E. Kroner, International Journal of Solids and Structures 3 (1967) 731.

19. Y.D. Ha and F. Bobaru, International Journal of Fracture, 162 (2010), 229. 
20. E. Madenci and S. Oterkus, Journal of the Mechanics and Physics of Solids, 86 (2016), 192.

21. E. Madenci and S. Oterkus, Engineering Fracture Mechanics, 175 (2017), 31.

22. J.T. Foster, S.A. Silling and W.W. Chen, International Journal for Numerical Methods in Engineering 81 (2010), 1242.

23. F. Baber, V. Ranatunga and I. Guven, Journal of Composite Materials (2018), p.0021998318774100.

24. B. Ren, C.T. Wu, P. Seleson, D. Zeng, and D. Lyu, International Journal of Fracture (2018), 1.

25. E. Celik, I. Guven and E. Madenci, Theoretical and Applied Fracture Mechanics 55 (2011), 185.

26. A. Shojaei, U. Galvanetto, T. Rabczuk, A. Jenabi and M. Zaccariotto, Computer Methods in Applied Mechanics and Engineering 343 (2019), 100.

27. C. Wright, M. K. Shah, L. Powell, I. Armstrong, Application of AFM from Microbial Cell to Biofilm, Scanning, Vol. 32, 134-149 (2010)

28. S.A. Silling, and E. Askari, Computers and Structures 83 (2005) 1526.

29. E. Madenci and E. Oterkus, Peridynamic Theory and Its Applications, Springer, New York, 2014.

30. H. Y. Hou, N. K. Chang and S. H. Chang, Nanomechanics of Materials and Structures, Dordrecht: Springer Netherlands, (2006) 171.

31. R. M. Overney, D. P. Leta, C. F. Pictroski, M. H. Rafailovich, Y. Liu, J. Quinn, J. Sokolov, A. Eisenberg and G. Overney, Physical Review Letters 76-8 (1996) 1272. 
32. J. H. Teichroeb, and J. A. Forrest, Physical Review Letters 91-1 (2003), 16104.

33. D. R. Askeland, and P. P. Pradeep, The Science and Engineering of Materials, Chapman and Hall, London, 1996

34. He, L., et al., Hyperthermal hydrogen induced cross-linking and fabrication of nanowrinkle patterns in ultrathin polymer films. Surface \& Coatings Technology, 2015. 261: p. 311-317.

35. Ao, Z.M. and S. Li, Temperature- and thickness-dependent elastic moduli of polymer thin films. Nanoscale Research Letters, 2011. 6.

36. Chung, J.W., et al., Biaxial elastic modulus of very thin diamond-like carbon (DLC) films. Diamond and Related Materials, 2001. 10(11): p. 2069-2074.

37. Birleanu C., Pustan M., The Effect of Film Thickness on the Tribomechanical, Properties of the Chrome-Gold Thin Film, (2016) Proceeding of Symposium on Design, Test, Integration \&Packaging of MEMS and MOEMS.

\section{LIST OF FIGURE CAPTIONS}

Fig. 1. A)Schemeatic of fabricated specimens for nanoindentation B) Scanning electroic microscope image of PS film on PDMS substrate.

Fig. 2. AFM surface profiles of A) PDMS substrate B) PS film edge on PDMS susbtrate.

Fig. 3. SEM image of the AFM cantilever used in nanoindentation process. B)Schematic of force and indentation calculations from the raw AFM data. 
Fig.4 A) Damaged and undamaged representation of a peridynamic bond B) Force-stretch relation for a peridynamic material interaction.

Fig. 5. Interaction of a material point with its neighboring points in Peridynamic model.

Fig. 6. Procedure to determine the reaction force on the indenter [29]

Fig. 7. Experimental force versus deformation results

Fig. 8. Comparison of force versus deformation results between experimental and PD Theory results on bulk PDMS sample.

Fig. 9. Comparison of the force vs. deformation results between experimental and PD Theory results on thin-film PS deposited on PDMS sample.

Fig. 10. Deformed configuration obtained by simulation using PD Theory on thin-film PS deposited on PDMS sample ((a) full view, (b) close-up view).

Fig. 11. Variation of elastic modulus as a function of sample thickness 


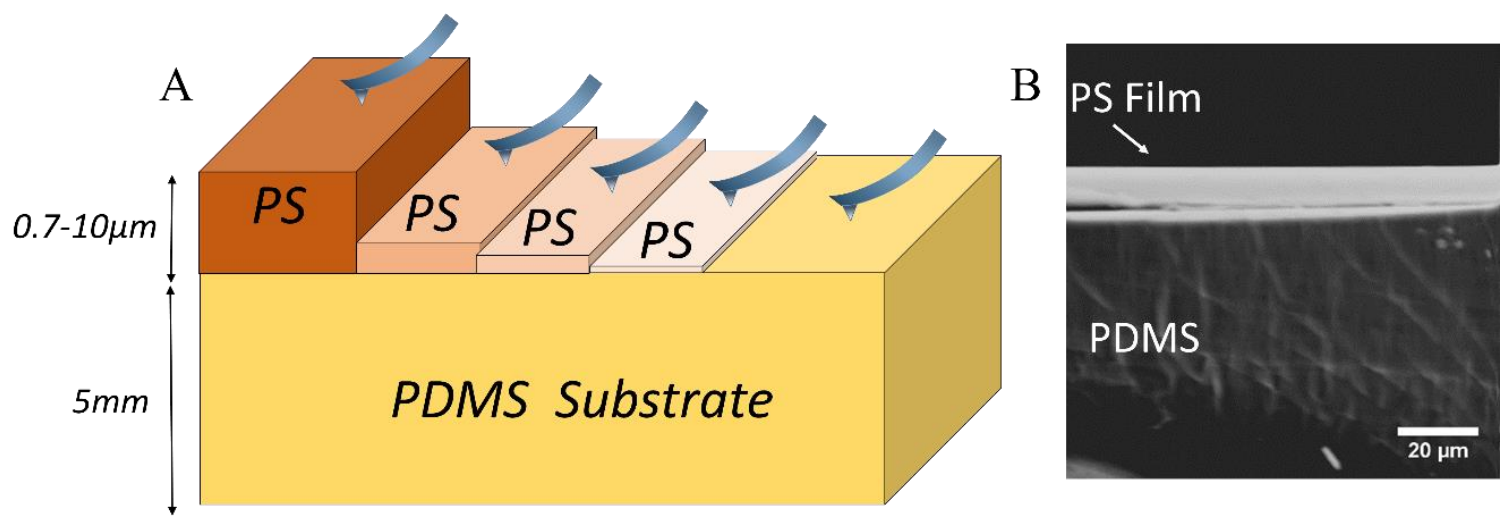

Figure 1. 

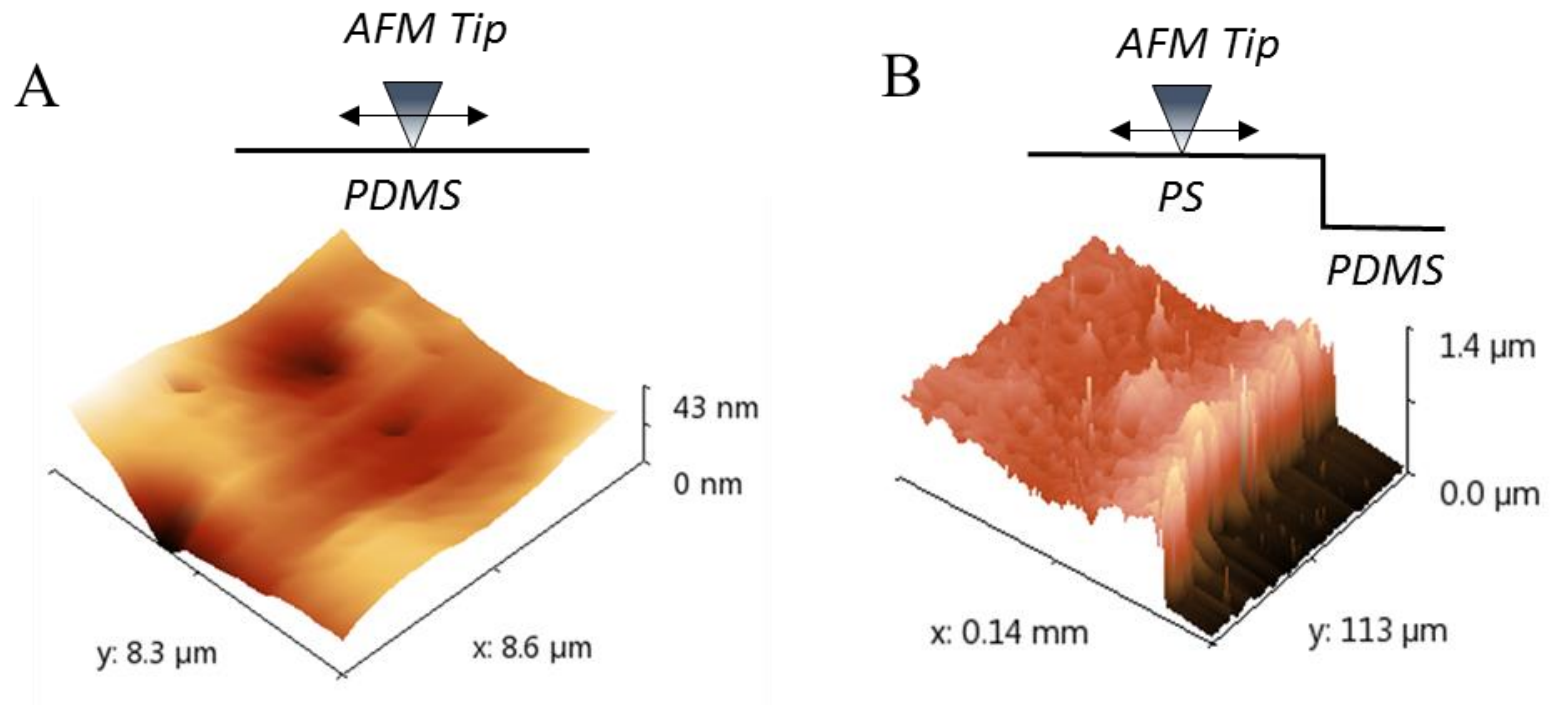

Figure 2. 

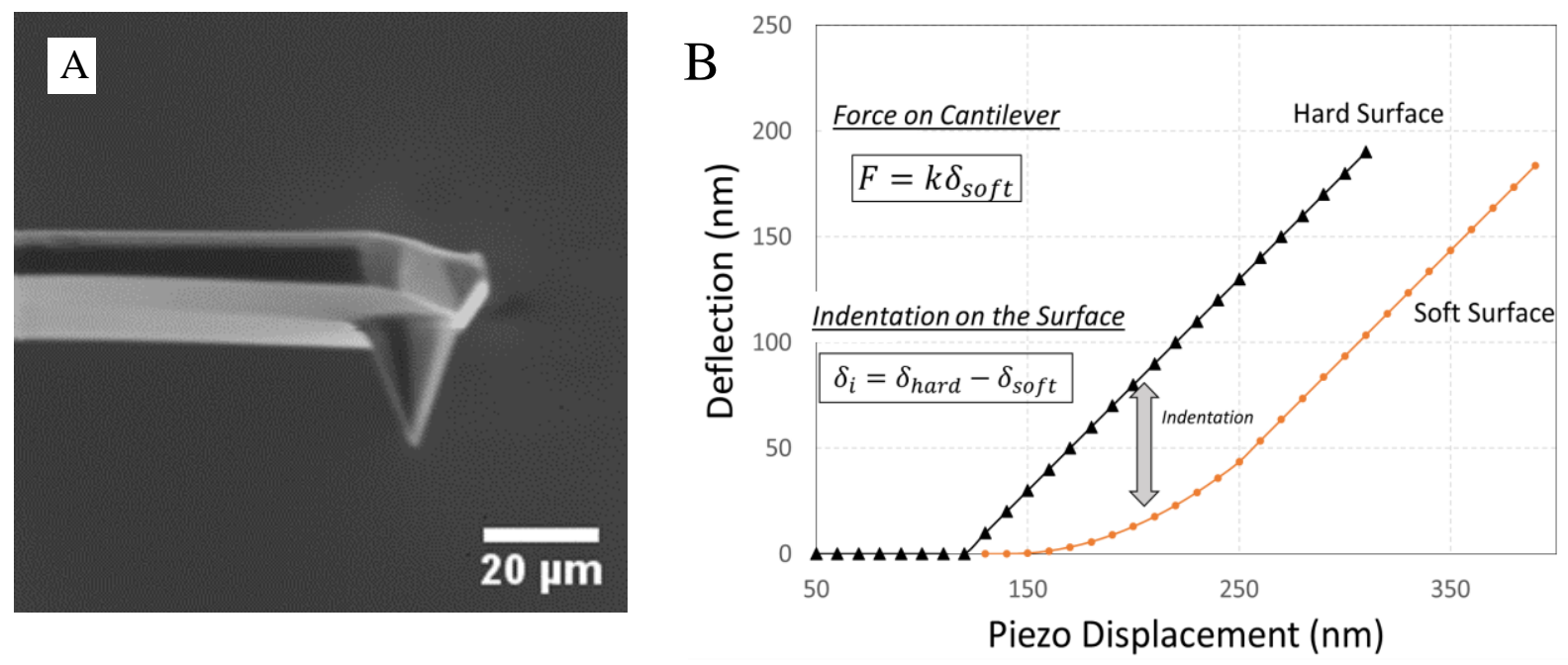

Figure 3. 

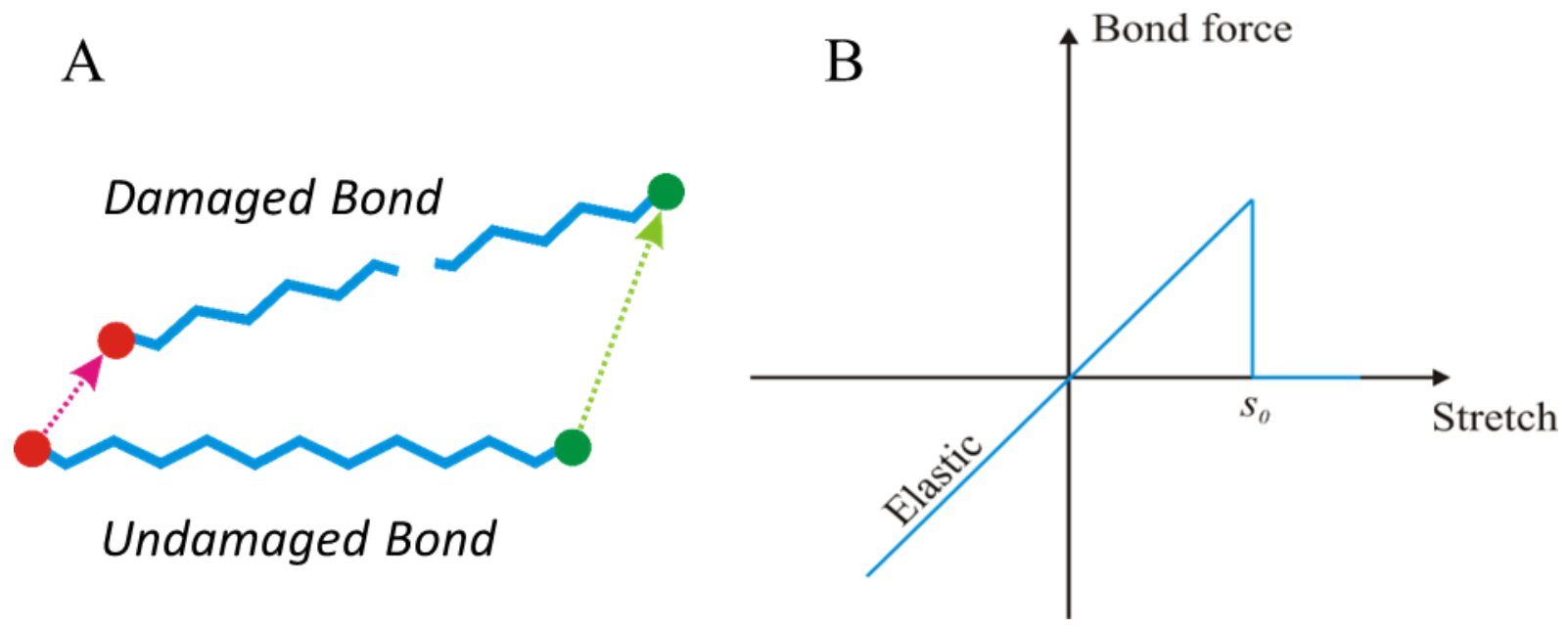

Figure 4. 


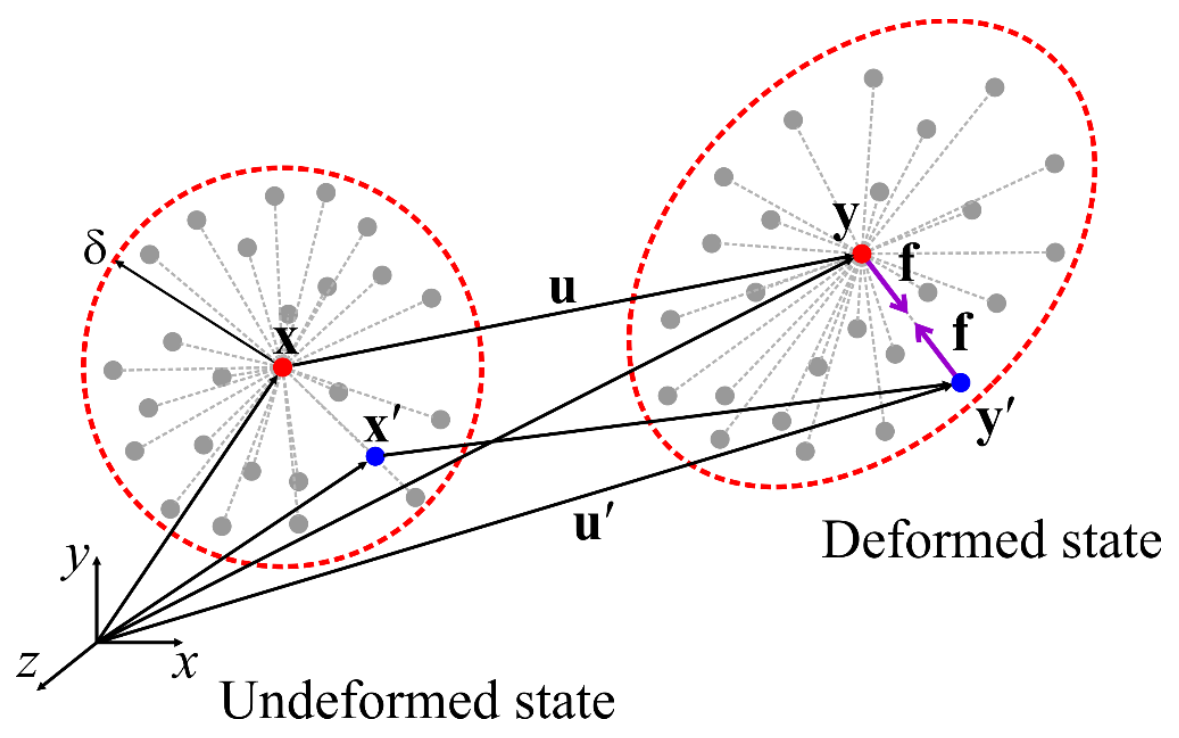

Figure 5. 


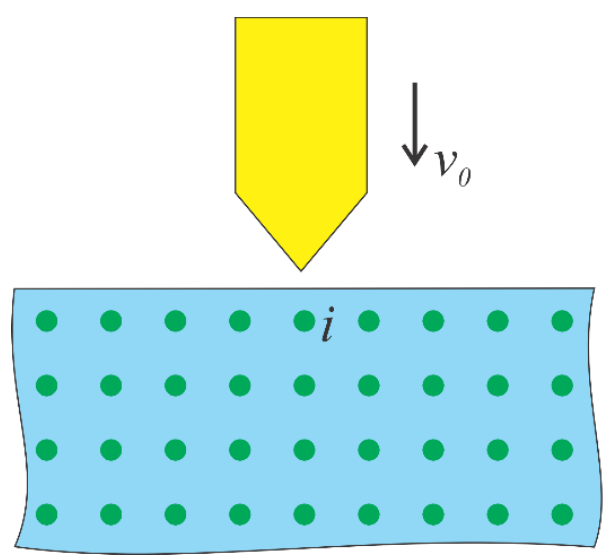

Time, $t$

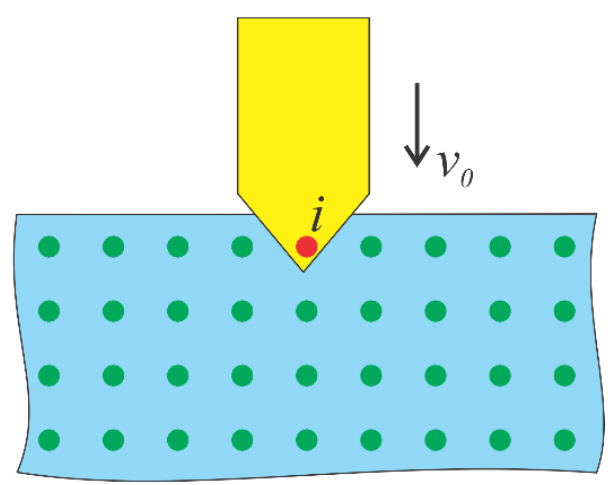

Time, $t+\Delta t$

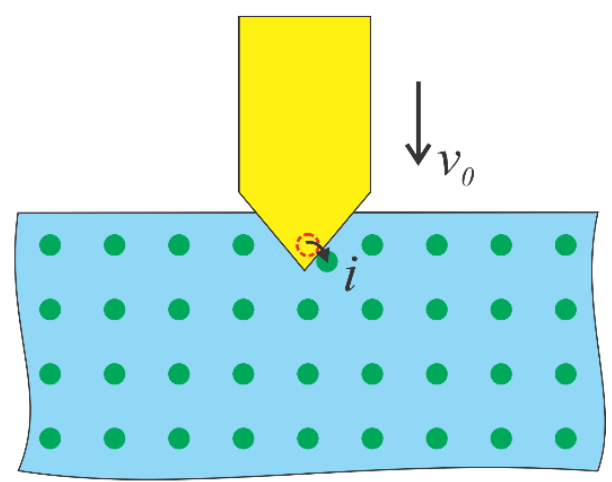

Time, $t+\Delta t$

Figure 6 


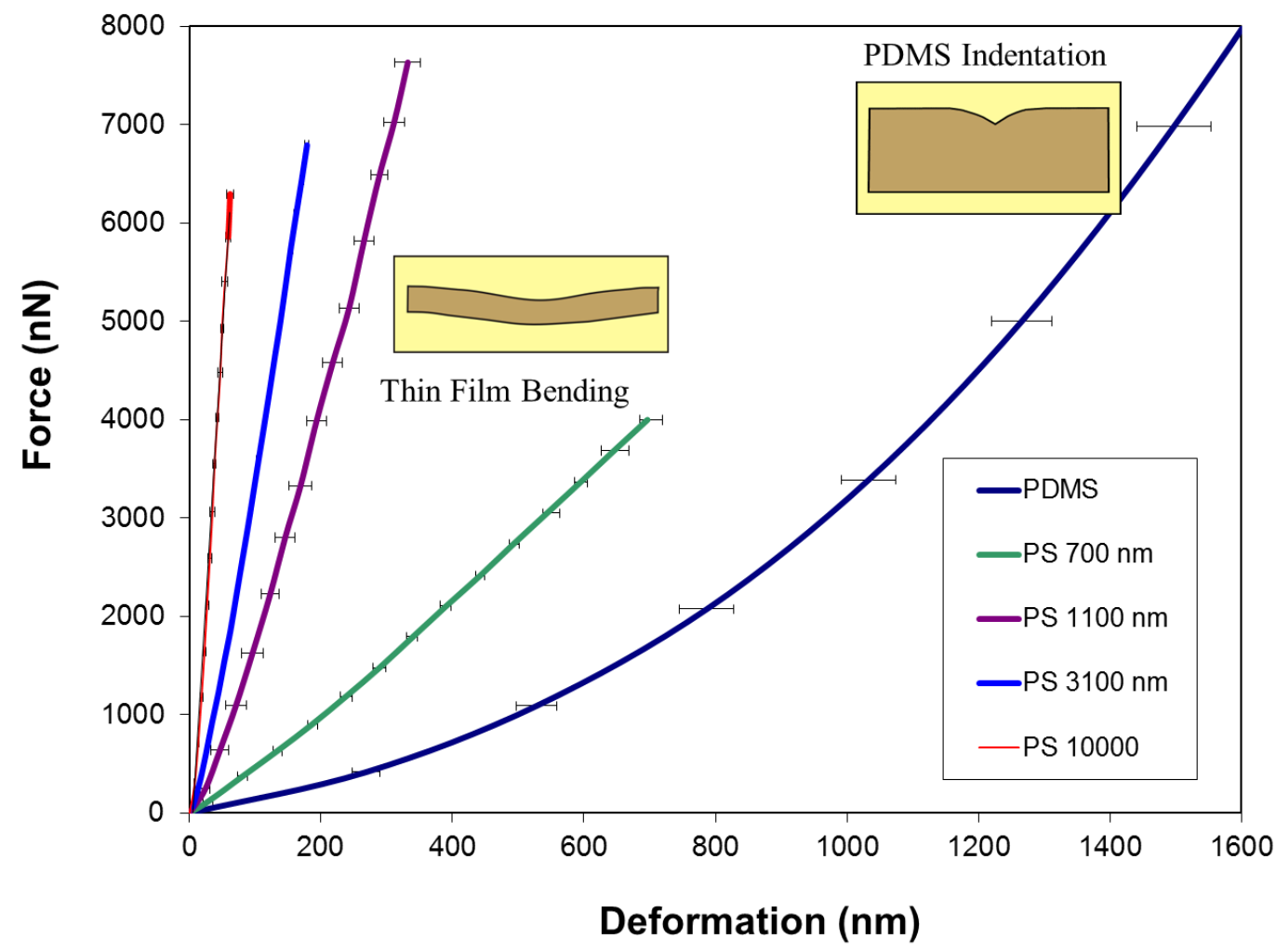

Figure 7. 


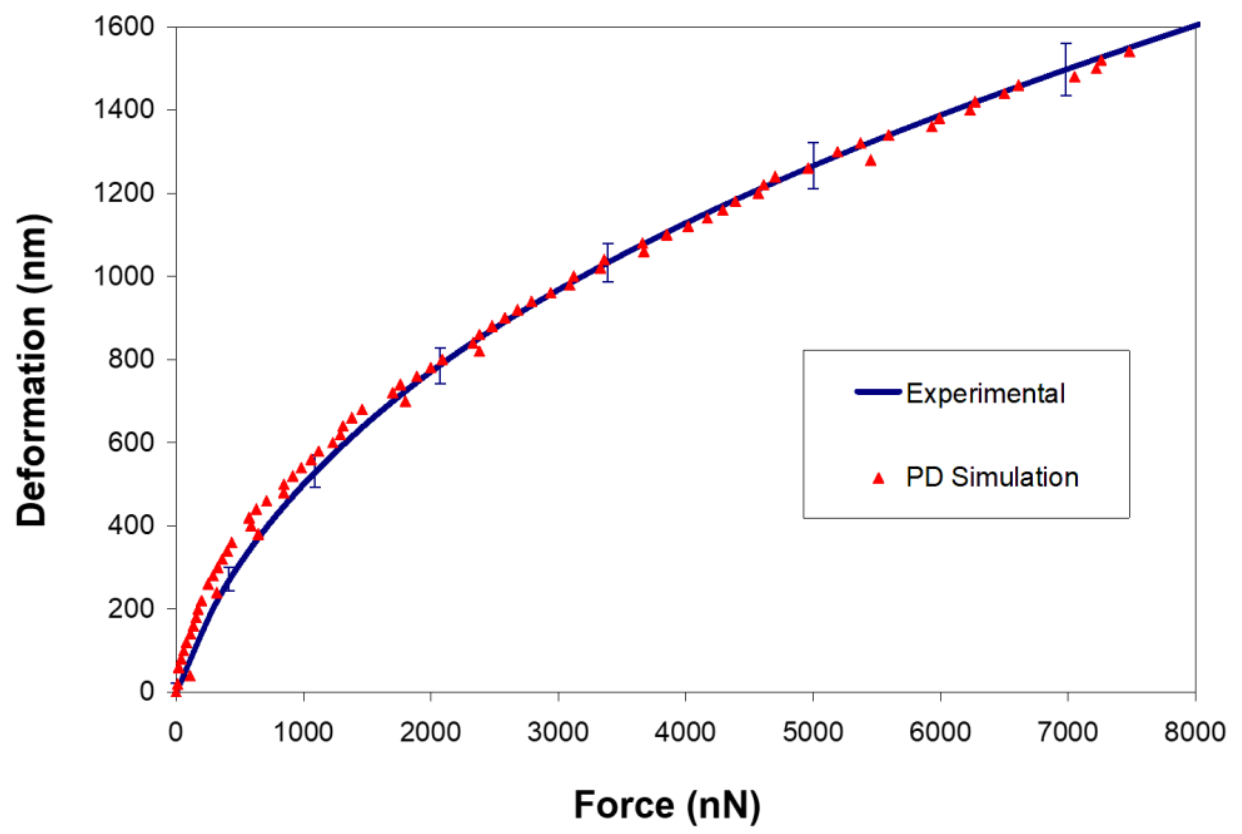

Figure 8. 


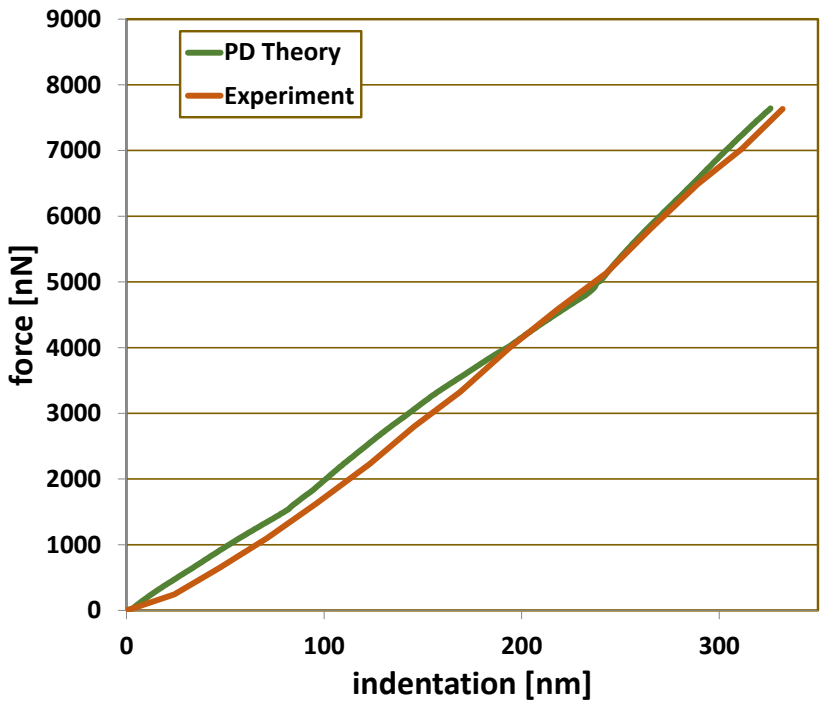

Figure 9. 


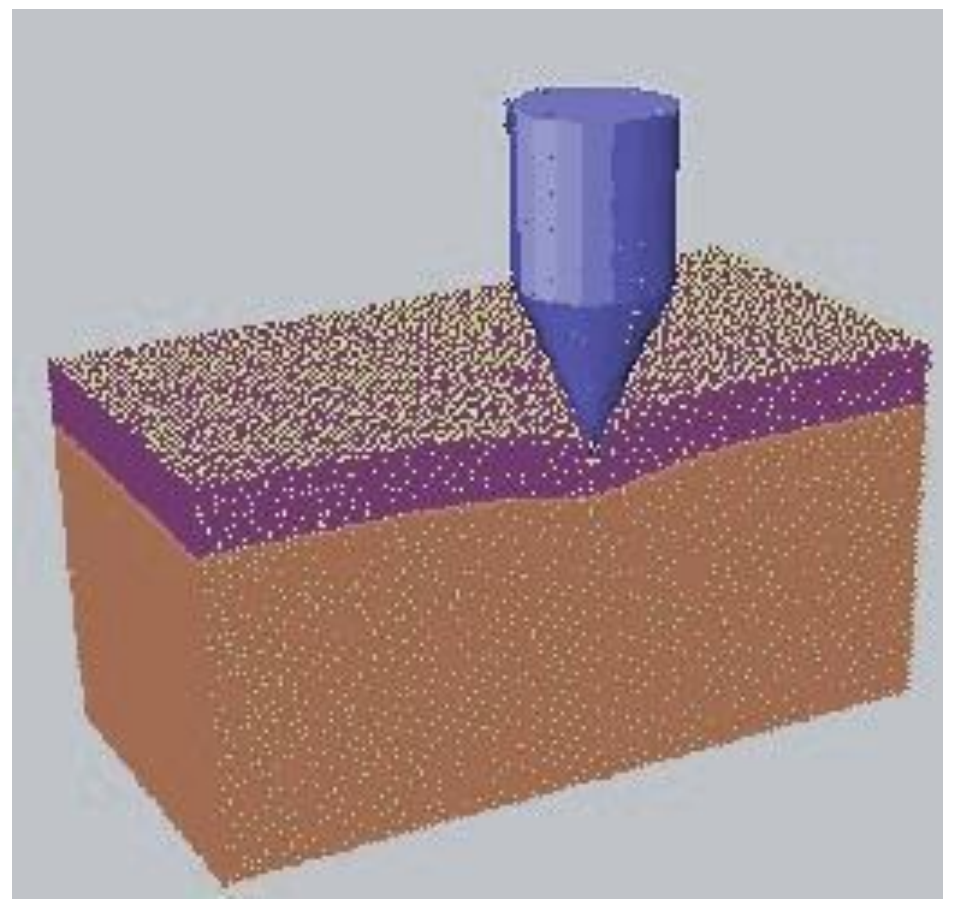

(a)

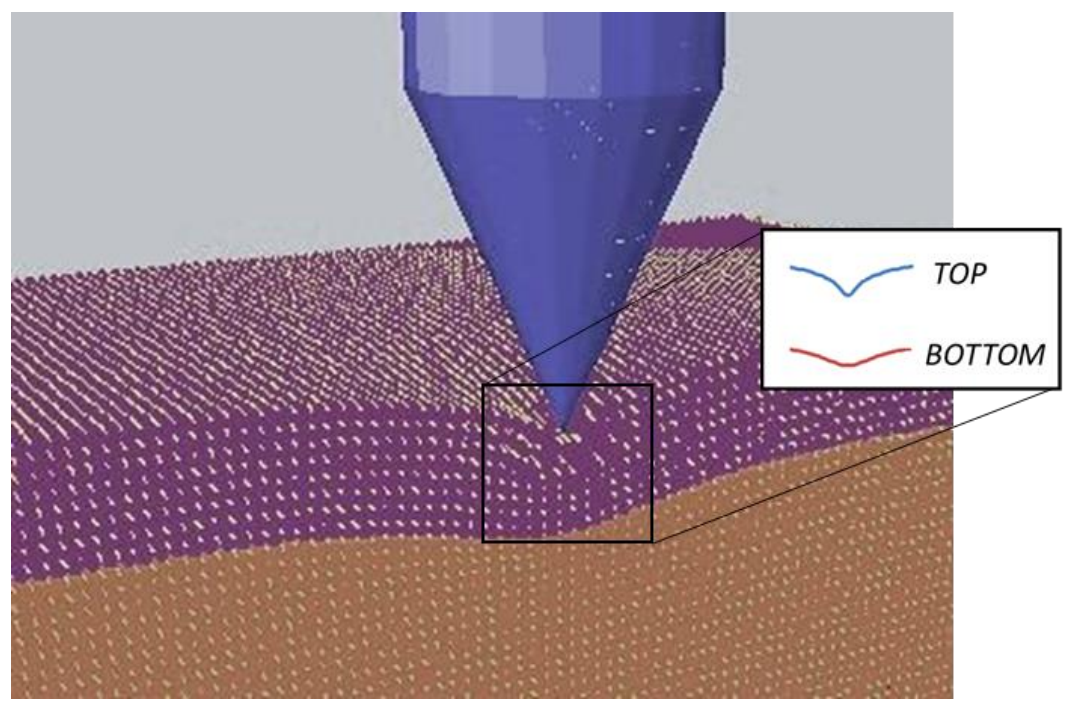

(b)

Figure 10. 


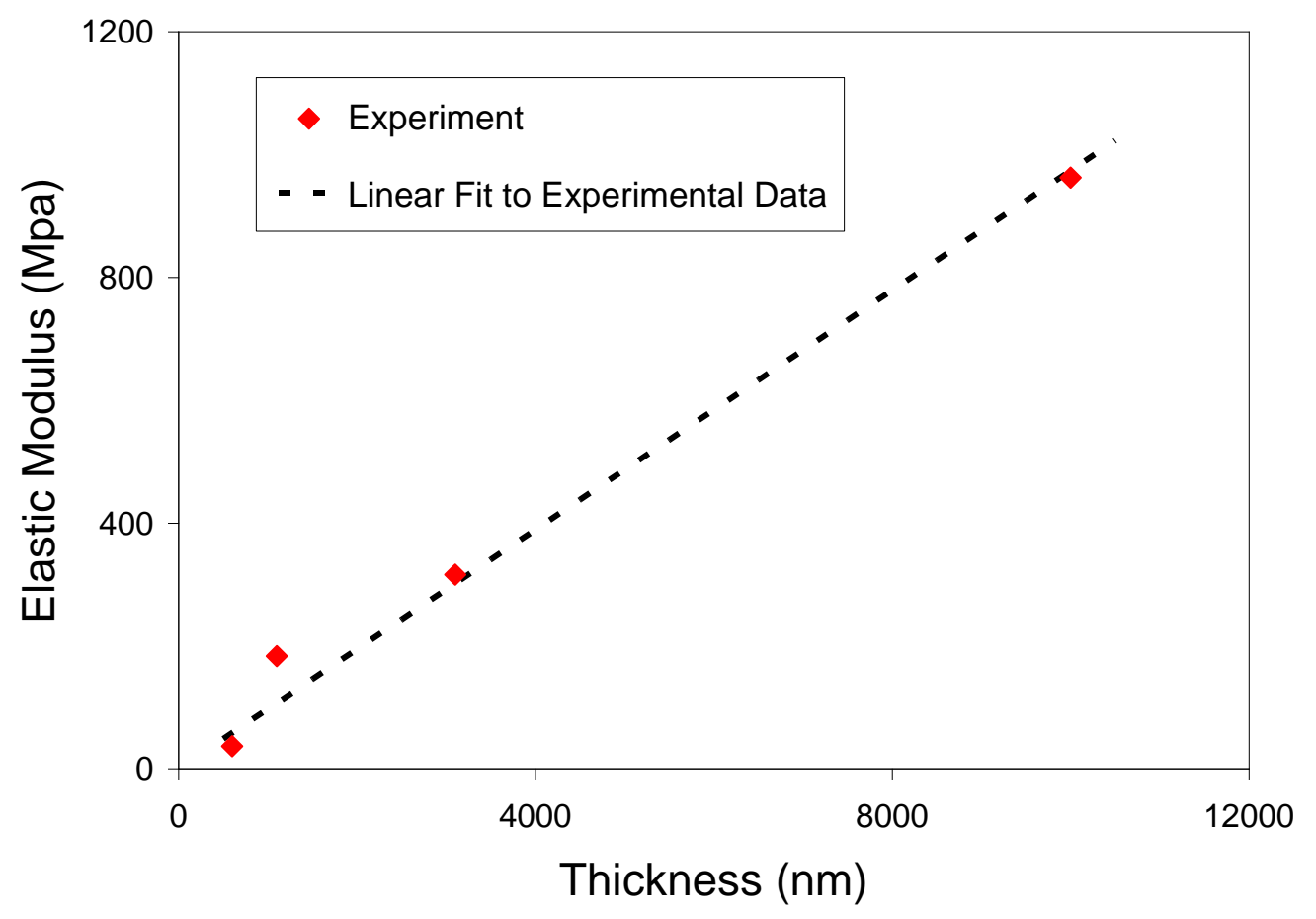

Figure 11. 